\title{
Investigation of PPLN waveguide uniformity via second harmonic generation spectra
}

\author{
Alan C. Gray, Sam A. Berry, Lewis G. Carpenter, James C. Gates, Peter G. R. Smith, and Corin B. E. Gawith
}

\begin{abstract}
Experimental data collection methods and a corresponding numerical model are presented to investigate the quality of waveguide fabrication in nonlinear optics. The method utilises white light interferometry and standard image recognition techniques to calculate a waveguide propagation constant function. This enables comparison of a numerical second harmonic spectrum in quasi-phasematched materials, such as periodically poled lithium niobate, with the waveguide's experimental phasematching spectrum. Using the presented method, a $3^{\text {rd }}$ order polynomial fit to waveguide ridge width is demonstrated to be in good agreement with experimental phasematching spectra. The presented technique provides a nondestructive route to discriminate between issues in fabrication steps in nonlinear waveguide design.
\end{abstract}

Index Terms-

\section{INTRODUCTION}

A CCESS to shorter wavelength devices and more efficient sources, which utilise high stability pump sources available at telecommunication or infra-red wavelengths, has driven research towards the advancement of nonlinear waveguides. Waveguides provide the potential for an increase in longitudinal spatial confinement between the pump and generated signal in comparison to bulk crystal focusing conditions developed in the early stages of nonlinear optics [1]. However, the numerical means to investigate the fabrication limitations in these waveguides has not been thoroughly examined.

In the limited literature in the area of numerical modelling of waveguide non-uniformity, Laurell and Arvidsson have discussed diffusion fluctuations in channel waveguides in birefringent phasematched lithium niobate (LN) [2]. They assume a parabolic propagation constant function to act upon their phasematching conditions with good experimental agreement. More recent work by Umeki et al.[3] assumes a sinusoidal function with an inverse dependence on waveguide length to conclude their bonded, thinned and diced periodically poled LN (PPLN) waveguides are close to optimal fabrication. Nevertheless, they clearly correlated the distortion of a nonlinear spectrum with a decrease in maximum conversion efficiency. Chauvet et al. also numerically investigated how a linear variation in waveguide width of their LN ridge waveguides could form two phasematching peaks in their experimental spectrum [4]. In addition, they calculated that a $200 \mathrm{~nm}$ width

Manuscript received June 5, 2019. This material is based upon work supported by the Air Force Office of Scientific Research under award number FA9550-16-1-0531, in part by the Royal Academy of Engineering Senior Research Fellowship RCSRF1718639, and in part by the Engineering and Physical Sciences Research Council (EPSRC) under grant EP/M024539/1. (corresponding author: Alan C. Gray.)

The authors are with the Optoelectronics Research Centre, University of Southampton, Southampton, SO17 1BJ, UK. (e-mail: A.C.Gray@ soton.ac.uk). variation would drop their conversion efficiency to one-third of its maximum potential. Furthermore, an elegant method is presented by Chang et al. [5] using frequency-resolved optical gating to study waveguide nonuniformities, but the method is only applicable to chirped period grating structures and pulsed pump sources.

Zinc-indiffused waveguides in channel or ridge geometries have been demonstrated as a route for guiding both orthogonal polarisation optical modes with high nonlinear conversion efficiencies [6], [7]. Advancing on current technology, we utilise ultra-precision dicing as a single-step process for waveguide and facet definition following the indiffusion of a planar zinc layer. The zinc-indiffused waveguides, used in this study, have been shown to be intrinsically resistant to optical damage [7].

Understanding fabrication imperfections and tolerances is not an area of research specifically for nonlinear generation in PPLN. Recent advancements in periodically poled potassium titanyl phosphate (PPKTP) waveguides typically utilise rubidium indiffused and ultra-precision dicing to fabricate nonlinear optical waveguides [8], [9], in a similar fashion to waveguides presented in this letter. Development of a method to provide feedback in the early stages of waveguide fabrication, to identify which processing step is a limiting factor, is the key purpose of this work.

\section{OPTICAL CHARACTERISATION AND NUMERICAL METHODOLOGY}

The work presented will utilise PPLN as the nonlinear crystal. The PPLN is fabricated by applying a patterned photoresist to the $-\mathrm{z}$ face of $\mathrm{z}$-cut, magnesium doped $\mathrm{LN}(\mathrm{MgO}: \mathrm{LN})$ followed by the application of an electric field greater than the material's coercive field via liquid electrodes. Domain reversal is achieved with a pre-defined period, $\Lambda$, of $2.2 \mu \mathrm{m}$ to satisfy phasematching conditions. Due to the short period, the grating width was limited to $200 \mu \mathrm{m}$ to prevent significant domain broadening and merging. Further details on the domain inversion process can be found in Wright et al.[10]. Waveguide fabrication is carried out through deposition of a metallic zinc layer followed by a diffusion step at $900{ }^{\circ} \mathrm{C}$ for $1 \mathrm{hr}$ on a $50 \mathrm{~mm} \times 10 \mathrm{~mm}$ PPLN chip, Fig. 1. This results in a planar waveguide which is single-mode in the vertical axis. The $\sim 7 \mu \mathrm{m}$-wide ridge waveguide and end facets are formed via ultra-precision dicing, similar to that presented by Carpenter et al. [11]. We perform the poling process prior to waveguide fabrication due to findings from Ming et al. [6] in which a reduction in poling quality was observed if the fabrication process flow was reversed. 


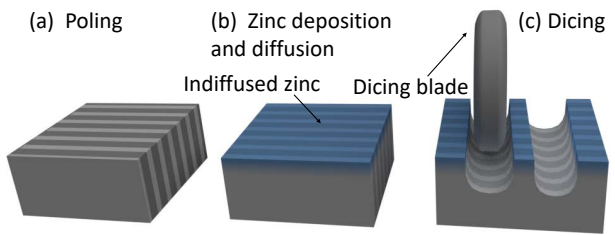

Fig. 1. Waveguides fabricated in this letter were prepared via (a) periodic domain inversion, (b) deposition and indiffusion of zinc followed by (c) ultraprecision dicing for waveguide and facet definition.

Short wavelength conversion to the blue/UV regime provides a good scenario for testing the limitations of the waveguide fabrication process as the waveguides are required to be narrower to operate in the single- or few-mode guiding regime. Optical characterisation is carried out using the setup detailed in Fig. 2. A tuneable $780 \mathrm{~nm}$ laser (Sacher Lasertechnik) with an optical chopper (OC) in the beam path prior to fibrecoupling with polarisation-maintaining fibre (PMF). The input coupling to the PPLN waveguide (PPLN:WG) is optimised via adjustable collimation (Thorlabs ZC618APC-B) and a focussing aspheric lens (AL1, Thorlabs C260TMD-B). The output is approximately collimated prior to prism-separation of the pump and signal beams. The $390 \mathrm{~nm}$ beam is directed to a silicon photodiode (PD, Thorlabs PDB210A) for lockin amplification (LIA, Stanford Research Systems SRS830). Low power conversion allows for study of the phasematching spectra without considering photorefraction effects.

Using a white light interferometer (WLI, ZeScope, Zygo Corp.), a top-down view of the $14 \mathrm{~mm}$-long ridge waveguide can be observed. An image spanning $0.25 \mathrm{~mm}$ is taken every $0.5 \mathrm{~mm}$. Due to the sharp image intensity contrast created via the the drop in imaging surface position, an edge detection (ED) algorithm (Canny method [12]) can be used to determine the waveguide's width for each pixel column; of which the first and final detected edges in that column's pixels are used to calculate ridge width. This limits the error which would occur from surface roughness being mistakenly assigned as an edge. An average width of each PPLN:WG image can be calculated after removal of erroneous data points; carried out by filtering of data outside one standard deviation of the distribution. These data points are typically due to the ED algorithm failing to detect an edge and the width is incorrectly calculated as the distance between a surface roughness point and one edge. Dicing depth is not considered as this always exceeds the diffusion depth and thus does not affect modal properties.

The PPLN:WG width data is used as the basis of creating a model in the commercial waveguide modelling software

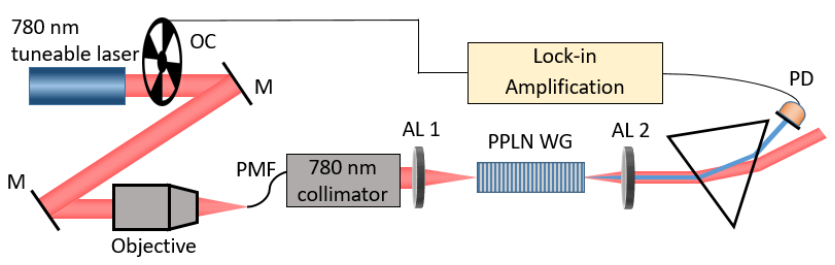

Fig. 2. Schematic of the characterisation setup for low-power nonlinear conversion for SHG of $780 \mathrm{~nm}$ laser light.
FIMMWAVE (Photon Design Ltd.) to extract an effective index value of the PPLN:WG at each point along the waveguide. A Gaussian diffusion profile is used to approximate the refractive index profile which creates the planar waveguide fabrication before dicing. We assume a maximum $\Delta \mathrm{n}$ of 0.003 , as per Ref. [7], and match the diffusion depth of the model to the evaluated, y-axis mode field diameter; this technique is detailed by Carpenter et al. [11]. As the relative nonuniformity is of key interest in this work, the propagation constant associated with each effective index value is offset as a relative propagation constant described by

$$
\delta \beta=\beta_{i}-\beta_{\min }
$$

where $\beta_{i}$ is the propagation constant for each ridge width modelled from the effective index, $\beta_{i}=2 \pi n_{\mathrm{eff}, \mathrm{i}} / \lambda$. Hence, the minimum $\delta \beta$ value is zero. Using similar mathematical notation with previous literature [3],

$$
\Delta \beta=\Delta \beta_{0}+\delta \beta(L) .
$$

This describes the phasematching condition utilised in the ordinary differential equations (ODE) governing signal generation in quasi-phasematched (QPM) $\chi^{(2)}$ nonlinear materials, discussed in detail by Roussev et al. [13]. In this work, second harmonic generation ( $\mathrm{SHG}$ ) will be used as the source of nonlinear signal spectra for numerical comparison. The function representing the numerically modelled $\delta \beta$ will be of key interest in this work. The distorted SHG spectrum which will be used for experimental comparison in this initial work is presented in Fig. 3. Maximum insertion loss of this waveguide is below $3 \mathrm{~dB}$. Assuming this is entirely propagation loss, the resulting change in phasematching spectrum will be negligible. Hence, propagation loss is not included in the model. Propagation loss is also known to affect only the bandwidth and efficiency of the phasematching spectrum and not to add asymmetry [14], which is our primary interest in this work.

A smoothed data set is also presented for ease of comparison with the numerical model. This was performed via removal of higher order frequency components, without loss of key spectral features. Additionally, an ideal $\operatorname{sinc}^{2}$ SHG is displayed

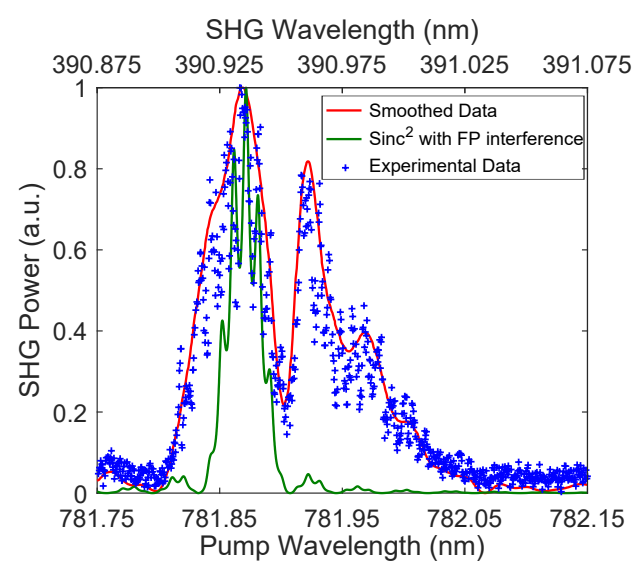

Fig. 3. Normalised experimental SHG spectra from the $\sim 7 \mu$ m-wide PPLN:WG and its smoothed curve. Also shown is a typical $\operatorname{sinc}^{2}(\Delta \mathrm{kL} / 2)$ with Fabry-Perot interference fringes for a $14 \mathrm{~mm}$ cavity. 


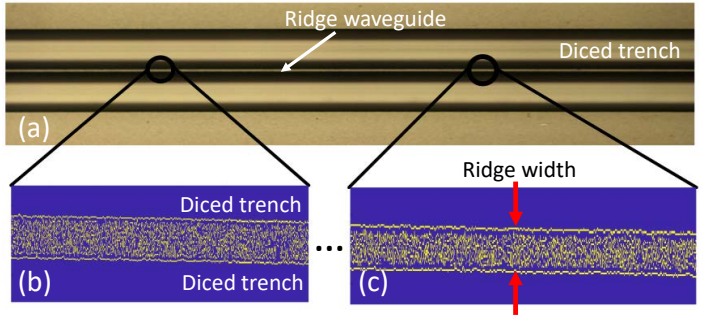

Fig. 4. (a) Optical microscope image of the PPLN waveguide. (b) and (c) Example data set resulting from edge detection of WIL images of the Znindiffused PPLN ridge waveguide. It can be seen that away from the ridge surface, no edges or surface roughness is detected and vivid edge lines are observed for calculation of local ridge width.

to represent the theoretical response of a perfect waveguide. This function details the effect a $14 \mathrm{~mm}$ Fabry-Perot cavity would have on the SHG spectrum. This is expected as the waveguide is not anti-reflection coated. It is evident from this that the main spectral features to be discussed in this letter are not dominated by the effect of Fabry-Perot interference.

\section{RESULTS AND DISCUSSION}

Examples of the edge detection data from sections of the ridge waveguide are presented in Fig. 4. The sharp intensity contrast between the waveguide surface and the adjacent diced cut, enabling the presented technique, is readily observed as a distinct lack of any edges in the diced trenches which define the ridge waveguide, shown in Fig. 1.

Fig. 5 represents the result from the WLI/ED data. The average ridge width on one end facet is compared with a calibrated optical microscope to validate the accuracy in the measurements and analysis. This discrepancy is typically $\sim 3 \%$ of the absolute value, providing further weight for the precision of the WLI/ED method. This discrepancy is then used as a calibration factor and applied to the remaining average thickness values along the ridge waveguide.

A polynomial fit to the average ridge width is used to form the required length dependant propagation-constant function, $\delta \beta$, in conjunction with FIMMWAVE to calculate the effective indices. This is shown in Fig. 6(a). We applied a $3^{\text {rd }}$ order polynomial fit to the data set as not to over-constrain the fit to features which would not be possible over small distances; due to the nature of the dicing blade's translation through the PPLN



Fig. 5. Ridge width data extracted via white light interferometry of the nonlinear waveguide designed for SHG of $780 \mathrm{~nm}$ laser light.

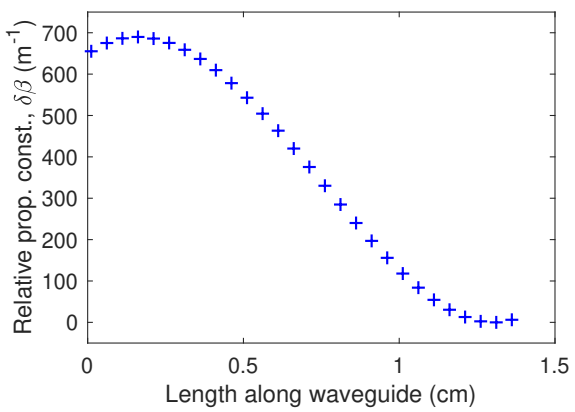

(a)

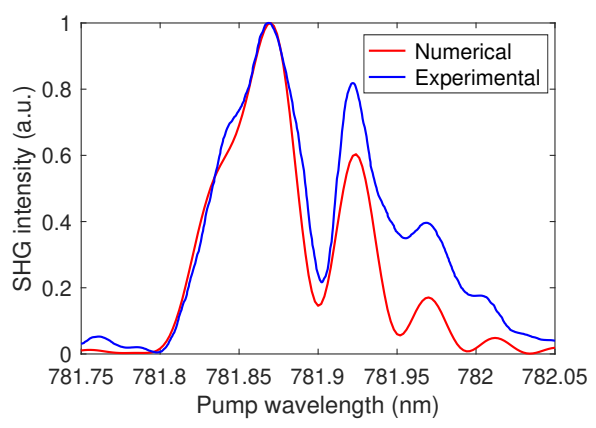

(b)

Fig. 6. (a) Propagation constant, referenced to zero, as a function of length along the waveguide. (b) Numerical SHG spectrum resulting from the $3^{\text {rd }}$ order polynomial fit of the waveguide width and the normalised, smoothed experimental data set from the LN WG designed for SHG of $\sim 780 \mathrm{~nm}$ laser light.

crystal. In addition, this is the lowest order fit to physically realise the effect of linear blade wear and the potential for wafer bow in both the $x$ - and $y$-axis which may arise from from conformation to the vacuum chuck. The phase mismatch, $\Delta \beta_{0}$ from Eq. 2, is calculated assuming bulk LN refractive indices [15], to align the spectrum in the wavelength axis, Fig. 6(b).

The numerical data presented in Fig. 6(b) has the correct features: lack of symmetry around the central peak; increased side lobe efficiency relative to central peak; and similar wavelength spacing of peaks. We expect the discrepancies in the data sets in Fig. 6(b) are likely due to the non-optimal poling quality, theoretically elaborated on by Fejer et al. [16] and has not been accounted for, particularly the contrast in minima. The zinc diffusion profile has also not been considered and is difficult to characterise experimentally.

To further utilise the presented method, we repeated the procedure for a $4 \mathrm{~cm}$-long waveguide which was designed for frequency doubling $1560 \mathrm{~nm}$ light to $780 \mathrm{~nm}(\Lambda=18.6 \mu \mathrm{m})$. This waveguide is fabricated using the same process presented in Fig. 1 but it is optimised for single-mode operation for guiding $1560 \mathrm{~nm}$ light in the vertical axis. However, the zinc diffusion process was carried out in a tube furnace with known poor thermal uniformity. This enables this waveguide to be used to investigate if the presented method is capable of quickly separating fabrication issues such as diffusion, i.e. waveguide depth variation, and ridge width variation. The SHG phasematching spectrum is presented in Fig. 7(b). The optical characterisation was carried out similar to Fig. 2, using a seeded erbium doped fibre amplifier and without LIA. 




(a)



(b)

Fig. 7. (a) Ridge width data resulting from the WLI/ED method of the $\sim 1560 \mathrm{~nm}$ doubling, $4 \mathrm{~cm}$-long waveguide. The waveguide is seen to have excellent ridge definition uniformity. Error bars represent $1 \sigma$ of the calculated ridge width for each WLI image. (b) Highly distorted sinc ${ }^{2}$ spectrum from the zinc indiffused LN waveguide designed for frequency doubling $\sim 1560 \mathrm{~nm}$ light and the associated model from data in (a).

The $\delta \beta$ value, as calculated by Eq. 1 and displayed in Fig. 7(a), is an order of magnitude lower than the data previously presented in Fig. 6(a); almost all average thickness data points fall within a single standard deviation of any data set. The range of the calculated average ridge width per image is $\sim 450 \mathrm{~nm}$. This concludes that the dicing fabrication step was precise to within the resolution of the metrology techniques.

The numerical model data presented in Fig. 7(b) is calculated using the same methodolgy as previously described. The distortion in the experimental data is evidently not caused by waveguide width variation and indicates another cause. Here it is expected to originate from waveguide depth variation from the diffusion process.

Although this waveguide was multimodal in the horizontal axis for both pump and signal modes, the phasematching of these higher order modes are at distinctly different wavelengths. For example, phasematching into the first-order (anti-symmetric) SHG mode was at $1562 \mathrm{~nm}$ and does not contribute to the spectra shown in Fig. 7(b).

This demonstrates the ability of the presented methodology to confirm and isolate, with relative ease, which fabrication step caused the distortion of the $\operatorname{sinc}^{2}$ SHG spectrum.

\section{CONCLUSiON}

The presented WLI and ED method has shown significant progress in enabling a numerical model to explain the distortion of nonlinear phasematching spectra than is currently available in literature. This technique takes advantage of the geometrical profile of ridge waveguides to generate a sharp contrast in images constructed from white light interferograms. Further advancements in the presented methodology lie in increasing the resolution of the WLI images. The presented method provides a route suitable for in-line, non-destructive testing of nonlinear waveguide fabrication including characterisation to assist in early stage process development. We have shown that this method is a route to quickly troubleshoot nonuniformities in multi-step fabrication processes for nonlinear ridge waveguides. Applications are primarily foreseen for diffused, ridge waveguides in either PPKTP or PPLN, currently at the forefront of blue/UV creation in nonlinear optical research.

\section{REFERENCES}

[1] G. D. Boyd and D. A. Kleinman, "Parametric interaction of focused gaussian light beams," J. Appl. Phys., vol. 39, no. 8, pp. 3597-3639, 1968.

[2] F. Laurell and G. Arvidsson, "Frequency doubling in Ti: $\mathrm{MgO}: \mathrm{LiNbO}_{3}$ channel waveguides," J. Opt. Soc. Am. B, vol. 5, no. 2, pp. 292-299, 1988.

[3] T. Umeki, O. Tadanaga, and M. Asobe, "Highly efficient wavelength converter using direct-bonded PPZnLN ridge waveguide," IEEE J. Quantum Electron., vol. 46, no. 8, pp. 1206-1213, 2010.

[4] M. Chauvet et al., "High efficiency frequency doubling in fully diced $\mathrm{LiNbO}_{3}$ ridge waveguides on silicon," J. Opt., vol. 18, no. 8, p. 085503 , 2016.

[5] D. Chang, C. Langrock, Y.-W. Lin, C. Phillips, C. Bennett, and M. M. Fejer, "Complex-transfer-function analysis of optical-frequency converters," Opt. Lett, vol. 39, no. 17, pp. 5106-5109, 2014.

[6] L. Ming, C. B. E. Gawith, K. Gallo, M. V. O’Connor, G. D. Emmerson, and P. G. R. Smith, "High conversion efficiency single-pass second harmonic generation in a zinc-diffused periodically poled lithium niobate waveguide," Opt. Express, vol. 13, no. 13, pp. 4862-4868, 2005.

[7] W. Young, M. M. Fejer, M. J. Digonnet, A. Marshall, and R. Feigelson, "Fabrication, characterization and index profile modeling of highdamage resistance $\mathrm{Zn}$-diffused waveguides in congruent and $\mathrm{MgO}$ : lithium niobate," J. Lightwave Technol., vol. 10, no. 9, pp. 1238-1246, 1992.

[8] C. C. Kores, P. Mutter, H. Kianirad, C. Canalias, and F. Laurell, "Quasiphase matched second harmonic generation in periodically poled $\mathrm{Rb}$ doped $\mathrm{KTiOPO}_{4}$ ridge waveguide," Opt. Express, vol. 26, no. 25, pp. 33 142-33 147, 2018.

[9] M. F. Volk, C. E. Rüter, M. Santandrea, C. Eigner, L. Padberg, H. Herrmann, C. Silberhorn, and D. Kip, "Fabrication of low-loss Rbexchanged ridge waveguides in z-cut $\mathrm{KTiOPO}_{4}$," Opt. Mater. Express, vol. 8, no. 1, pp. 82-87, 2018.

[10] T. A. Wright et al., "Two-way photonic interface for linking the $\mathrm{Sr}^{+}$ transition at $422 \mathrm{~nm}$ to the telecommunication C band," Phys. Rev. Appl, vol. 10, no. 4, p. 044012, 2018.

[11] L. G. Carpenter, S. A. Berry, R. H. Bannerman, A. C. Gray, and C. B. Gawith, "ZnO indiffused $\mathrm{MgO}$ : PPLN ridge waveguides," Opt. Express, vol. 27 , no. 17 , pp. $24538-24544,2019$.

[12] J. Canny, "A computational approach to edge detection," in Readings in computer vision. Elsevier, 1987, pp. 184-203.

[13] R. V. Roussev, C. Langrock, J. R. Kurz, and M. M. Fejer, "Periodically poled lithium niobate waveguide sum-frequency generator for efficient single-photon detection at communication wavelengths," Opt. Lett. vol. 29, no. 13, pp. 1518-1520, 2004.

[14] Y.-R. Shen, "The principles of nonlinear optics," New York, WileyInterscience, 1984, 575 p., 1984.

[15] O. Gayer, Z. Sacks, E. Galun, and A. Arie, "Temperature and wavelength dependent refractive index equations for $\mathrm{MgO}$-doped congruent and stoichiometric $\mathrm{LiNbO}_{3}$," Appl. Phys. B, vol. 91, no. 2, pp. 343-348, 2008.

[16] M. M. Fejer, G. Magel, D. H. Jundt, and R. L. Byer, "Quasi-phasematched second harmonic generation: tuning and tolerances," IEEE J. Quantum Electron, vol. 28, no. 11, pp. 2631-2654, 1992. 